\title{
Epistemological Issues in the Making of an African medicine: Sutherlandia (Lessertia Frutescens)
}

\author{
Olajide Oloyede \\ Research in Anthropology and Sociology of Health (RASH) \\ Department of Anthropology and Sociology \\ University of the Western Cape, Cape Town \\ E-mail:jide.oloyede@gmail.com
}

\section{Abstract}

The scientific clinical trial of the African traditional medicine, Sutherlandia (Lessertia frutescens) forms an interface between the indigenous local knowledge of people living with Aids, traditional health practitioners and that of science and global health. Up till now, no cross-disciplinary studies have been done on epistemological questions concerning especially the knowledge and understanding of 'proof' of efficacy concerning an African traditional medicine. This paper draws together insights and analysis from the anthropology and sociology of health and healing in discussing the intersecting fields of knowledge and experience of pharmacology, phytotherapy and related fields, as well as that of biomedical and traditional health practitioners, and of research subjects involved in the making of clinical trials of sutherlandia (lessertia frutescens) in South Africa.

Keywords: South Africa, African medicine, bio-medicine, sutherlandia (lessertia frutescens) clinical trials.

\section{Introduction}

Traditional herbal medicines, generally considered as naturally-occurring plant-derived substances with minimal or no industrial processing that have been used to treat illness within local or regional healing practices (Cravotto et al, 2010) receive significant attention in global health debates. This attention seems, of recent, to centre more on randomised clinical trials (RCT) of these medicines, which still remain the prevailing source of healing in most part of Africa with an estimated $80 \%$ of people using them. ${ }^{1}$

Generally, the standard call is for evidence-based research of herbal medicines in relation to quality, efficacy and safety, or in terms of well-defined close/effect relationships. The supporting evidence that is needed revolves chiefly around well-controlled clinical trials including placebo-controlled trials, where appropriate and, bioavailability and 
pharmacokinetic studies. Such trials tend to receive generous funding from governments, pharmaceutical companies and herbal entrepreneurs. For example, China, India, Nigeria and the US are said to have made substantial investments in traditional medicine (WHO, 2002). South Africa made the provision for the investigation of traditional medicines (Pefile, 2005) in its national drug policy, and, in several African countries, there is an active promotion of traditional medicines for the treatment of HIV and associated symptoms by Health Ministries (Morris, 2002, Mills et al, 2005).

However, as the ever-expanding literature shows, randomised clinical trials of traditional herbal medicines remain controversial especially its underlying assumptions: the power and superiority of its mode of evaluation of efficacy. Some consider clinical trials as epistemologically problematic rejecting the notion that herbal medical treatment can be evaluated by scientific methods (Mackenzie-Cook, 2006). The premise for this is that science-based medicine is considered reductionist, whereas traditional herbal medicine is vitalistic (Coulter and Willis, 2004) and as such, it is problematic to evaluate the efficacy of traditional plants according to the criteria of evidence-based science. In addition, efficacy and safety are often evaluated "with different point of views and filtered by different opinions according to the clinical or traditional experience in the various folk medicines in different European countries" (Calapai, 2008: 428 citing Mahady, 2001). These observations, one may argue, remain in the tradition of the ancient ruminations on the extent to which medicine is a science and an art, a duality which Montgomery, in her recent volume How Doctors Think - Clinical Judgment and the Practice of Medicine, noted as a reminder that medicine remains poorly defined. Others, who stress the politics in this medical epistemology, see it as an extension of the politics of knowledge domination by the West through the strengthening of the long-standing 'hegemony' of bio-medicine.

At the heart of this strengthening 'agenda' is the special room given to traditional herbal medicines - complementary and alternative medicines (CAM), a term that is considered as reflecting the hegemony and indeed suggests hierarchies of knowledge. An opposing view, of this politics of knowledge, is what can be termed the 'preservation stance'. In this view, it is held that indigenous medical knowledge must be protected and preserved and organisations such as the Dakar (Senegal)-based Association for the Promotion of Traditional Medicine (PROMETRA) dedicates itself to preserving and restoring African traditional medicine and indigenous science. There are those who disagree with this view dismissing traditional medicines on the ground that it is based on 'superstition and neocremancy', suggesting instead that herbal medicines should be 'co-opted' into bio-medical knowledge and researched for this purpose as they "would not only improve and demystify its therapeutic qualities" (Nyika, 2007: 25), but "provide validated information to traditional healers and patients on" their judicious use (Mills, et al, 2005: 1). Such a view, one must hasten to add, seeks to standardise and control that which hardly falls within biomedical modality of knowing. 
In the pages to follow, the above issues are discussed within the general theme of knowledge, healing and medicinal plants drawing together insights and analysis from anthropology and sociology of health and healing. The literature on this is verse and it is not the objective here to attempt to cover the topics nor provide an authoritative review of the debate; my task is simply to present a relatively sustained examination and discussion of the more salient ones - the increasing clinical trials of herbal medicines as part of the 'movement' to give it a science base; the ethos of healing of traditional medicine and its incompatibility with the ethos of bio-medicine. The objective is to bring to the fore the dilemmas, difficulties, challenges in the quest for the treatment of chronic diseases in a context in which the vast majority rely on traditional herbal medicine but science and scientific methods are accepted as the major foundations for health care and biomedical knowledge inform health policy. The key point is: clinical trials' 'logic of refutation' would seem to provide, epistemologically, a partial knowledge of the efficacy and safety of traditional medicines based strongly on the traditional healing's 'logic of affirmation', which is readily dismissed by the former. Both require rigorous examination in the quest to establish a plant's efficacy; defining all potential warrants for medicinemaking in clinical trials strengthens the efforts to structure hierarchies of knowledge that denote and devalue knowledge derived from anything other than rigorously conducted clinical research. It is an old call but given that there has not been any intellectual resolution that can serve as a platform on which biomedicine/traditional healing can jointly proceed to achieve optimal practice of medicine in the context described earlier, the current piece aims, modestly, to stimulate further philosophical inquiry for that purpose. The discussion draws also from an on-going 'conversation' with traditional health practitioners in a UWC-PEPFAR project ${ }^{2}$ that brings together bio-medical and traditional health practitioners in dealing with HIV/AIDS as well as from phytotherapy, considered as a complementary approach to healing and preventing disease.

The paper is an outgrowth of an ongoing study of the philosophy and environment of clinical trials of sutherlandia. The discussion is structured as follows: the first section deals with herbs and the scientific testing of their safety and efficacy in the form of clinical trials. This is followed by the section in which traditional medical health system is discussed, specifically, its knowledge base. These sections set the context for a discussion of an African herbal plant, the sutherlandia, and the clinical trial of this plant is examined against the issues raised in the sections. These are all brought together in the last section which raises some questions with the purpose of showing the complexities of the making of a medicine in a context where most patients utilize the services of traditional healers.

\section{Herbs for medicines and clinical trials of herbs}

Generally, herbs can be any form of a plant or plant product including 
leaves, stems, flowers, roots and seeds. As such they are often perceived as 'natural' and thus somehow safe (Ernst, 2002). These plants can either be sold raw or as extracts, where they are mace rated with water, alcohol or other solvents to extract some of the chemicals. The resulting products contain dozens of chemicals, including fatty acids, sterols, alkaloids, flavonoids, glycosides, saponins, and others (Bent, 2008). However, many different side effects to herbs have been reported and recently reviewed (De Smet, 2002). A common toxity to herbal medicines involve pyrrolizidine, alkaloids, which are complex molecules found in certain plants that may be used or inadvertently added to herbal medicines. These alkaloids produce hepatotoxity through a characteristic veno-occulsive disease that may be rapidly progressive and fatal (Stickel, Patseuker and Scuppan, 2005). Yet, herbal medicines are reported to have no-side effects except in cases where the herbs are used with synthetic drugs. Some herbs, as De Smet (2002) notes are known to interact with pharmaceutical drugs. St John's wort, for example, is said to be the most notoriously interactive herbal product, and has been shown to interfere with numerous drugs metabolized by the cytothrome P-450 liver enzyme system, including protease inhibitors, chemotherapeutic agents and oral contraceptives (see, for example, Nebel et al, 1999; Prost et al, 2000). Some authorities note that many herbs, including Kava, Valerian and St John's wort, have the potential valerian to interact with anesthetic agent $s$ and other drugs given in the perioperative period (Ang-Lee, Moss, Yuan, 2001). One must, however, state that the true frequency of side effects for most herbs is not known because they have not been tested in large clinical trials and also because surveillance systems are much less extensive than those in place for pharmaceutical products (Bent, 2008).

Some researchers consider herbal medicines as not fulfilling the requirements of well established medicinal use despite the long tradition of use. The adverse effects of phytotherapeutic agents are less frequently compared with synthetic drugs; however, well controlled clinical trials and numerous individual case reports have now confirmed that such effects do exist (Calixto, 2000). One cannot rule out the fact of the longstanding use of herbal medicines as a guarantee of their safety. At the same time, one cannot say that because of this, there safety and efficacy cannot be questioned. The question therefore is: what is the level of acceptable safety and efficacy? This question raises a general problem: the evaluation of efficacy. According to what criteria? Generally and principally, as has become quite known, it is according to the criteria of evidence-based medicine. As suggested by Calapai and Caputi (2007), the efficacy of medicinal plants can be tested in clinical trials much like synthetic drugs, although numerous methodological and logistical problems exist. The methodological issues include research design. There is a consensus that all human subjects research must 'maintain valid study designs', however, the characteristics of a valid research design would seem to be an issue. There is the argument, especially by those trained in biomedical methods of clinical investigation that the only valid source of knowledge regarding clinical efficacy must come from one 
type of research design: the randomised blind, placebo-controlled trial (Tilburt and Kaptchuk, 2007).This is considered a gold-standard and any deviation from it makes the research worthless.

There is an opposing view which suggests that the attempt to evaluate traditional therapies with biomedical methodologies, may fail to generate true knowledge since that knowledge itself depends on a scientific vocabulary that only makes sense within the concepts of biomedicine (Schaffner, 2002; Fabrega, 2002).They suggest that the "standard notions of experimental design criteria represents an imperialistic 'western' mode of thinking" (Schaffner, 2002, p7). Kaptchuk (2001) suggested that research on herbal medicines should typically employ experimental research designs such as the RCT, even if research tools are not perfect; they are, in fact considered the best methods for furthering knowledge. What we, therefore, have is that clinical trials of herbal medicines continue with its tradition of 'trials' - science's tradition. The trials maintain focus on safety and efficacy hardly showing interest in the cultural context of the use of such plants. What is the cultural context of the use of such plants? Of what benefit is the cultural context to the issue of efficacy and safety? In asking these questions, one is invariably asking questions of the knowledge base of traditional medical practice. What is this knowledge base? The answer is well-known as so much has been written; what can be done for the purpose of the discussion at hand is to present the core. This is done within the context of what can be considered as the traditional health system and this follows immediately.

\section{Traditional Medical Practice and its knowledge base}

Forster's (1976) notion of 'personalistic' serves as a useful starting point in describing the traditional medical system. In such a system, illness and misfortune can be caused by the purposeful manipulation of supernatural powers so that the religious and medical systems are intermeshed. Personalistic etiologies are based on the idea that the volition or the intervention of an extra-natural force causes misfortune. The treatment of personalistic illness is the specialty of the traditional healer who conducts healing ceremonies aimed at appeasing angered gods or spirits, or counteracting the influence of witches or others who possess the power to cause illness. On this point, one needs to point out that in as much as a deity, a human witch or sorcerer, or non-human agent such as an evil spirit or ancestor can use powers beyond those of everyday experience to harm living human beings or control their behaviour, the converse applies in that the same powers can be proactive and assure success in life to those who possess them. Given this, the medical practitioner in this system, referred to, generally as the traditional healer is "someone who is recognised by the community in which he \{she\} lives as competent to provide health care by using vegetable, animal and mineral substances and certain other methods based on social, cultural and religious background 
as well as the prevailing knowledge, attitudes and beliefs regarding physical, mental and social well-being and the causation of disease and disability in the community" (WHO, cited in Pretorious et al 1993). Obviously if such a person is responsible for disease and disability, then, he is a sorcerer, which then suggests that a sorcerer in this system is also considered a medical practitioner. This explains why the African traditional medical practitioner is various referred to as herbalist, native doctors, native healer, traditional doctor, medicine man, witch doctor, among others.

However, whether a healer or a sorcerer, a medical practitioner is an agent who is knowledgeable in culturally determined roles of procedure that can be used to control supernatural powers to bring about predetermined effects for self or client. The assumption is that these forces are part of a coherent interconnected universe in which acts of magic can project specific forces along pathways or connections between things to bring about desired goals. When there is interference with the cause of natural forces which results in action contrary to their 'natural programme', we can then say that there has been sorcery as Stevens (1996) also pointed out. It is important to note that Stevens (1996: 723) conceptually separate the forces of nature that can be magically programmed for a specific action from the powers of the anthropomorphised deities, ancestors, ghosts and spiritual beings that also inhabit the universe. Medical practitioners in personalistic systems are skilled in controlling natural forces and some also can capture the services of spirits. This is part of their very rich repertoire in preparing medicine. Included in this repertoire in some cultures are human images molded or carved from natural materials and used as conduits to channel the powers of nature toward cultural goals of 'magical mimesis' which, according to Taussig (1992), involves "both copy and substantial connection, both visual replication and material transfer" (Taussig, 1992: 8-14).

The making of medicine in this system is thus one in which the entire natural world is a source of power. Practitioners, therefore, incorporate plant and animal parts and other natural objects, as well as artefacts in varying combinations to draw upon the unique powers of each. The parts of plants and animals used tend to be what Rubin (1989) described as "signature elements". These include the leaves, roots of the plants, bark as earlier mentioned. In cultures where human figures are used as part of the making of medicines, such figures are more than mere imitations. As Wolff (2000) keenly observed, in the case of the place of human figures in the medicines of the Yoruba of South-western Nigeria, such figures are transformers that embody and channel the powers of the natural materials from which they are fashioned. In other words, he adds, enthusiastically, that the creation of the medicine figure is an act of embodiment with the intention of concentrating powers of nature for social goals. Most of these medicine figures must be further empowered, which was also noted by Wolff, before they fulfill their instrumental function. This can either be through putting them in contact with the additional magical ingredients and/or by having powers called into them through sacrifice and incarnations. 
There are other similar practices in sub-Saharan Africa. Wolff (2000) notes that, one of the best documented traditions of the medical use of human images as power objects, is that of the Kongo. He writes that Kongo medical practitioners make minkisi figures with magical ingredients inserted into wood or applied as thick mantles to the surfaces. It is known that such figures, when carved in human form, can be charged with positive curative or protective forces or conversely with destructive powers to bring misfortune or disaster to enemies. We see this also in the role of Esu in Yoruba medicine (Oloyede, 1999). As Wolff (2000) was correct to point out, in the larger inventory of the Yoruba sculpture in which the human figure is the leitmotif, the carved and modeled human images in indigenous medicines form a small and obscure category. However, their use in making medicines is huge; yet, it is herbal plants that are central in making medicines. In this regard, traditional healers are skilled botanists and have great talent for locating plants with medicinal properties.

In the conversations with traditional health practitioners in an ongoing research that was referred to earlier, they point out that their preference for sourcing the plants remains the village groves, jungles and the generally wild. This is based upon the belief that wildly collected materials are more effective than planted ones. This point is very crucial in the making of herbal medicines. Interestingly, scientific studies, seem, to some extent to support this assertion. Medicinal properties in plants are due mainly to the presence of secondary metabolities that plants need in their natural environments under particular conditions of stress and competition, which perhaps would not be expressed under monoculture conditions. Active ingredient levels can be much lower in fast growing cultivated stocks. In wild populations, plants can be older due to slow growth rates and can have higher levels of active ingredients. This scientific explanation is culturally explained by traditional health practitioners as simply part of the healing power of nature and only those trained who possess the skill and knowledge are directed to the plants in their natural habitat. In fact most personalistic illnesses can be treated only by traditional healers because they know the proper rituals part of which include gathering the appropriate herbal plants. While one can presume that cultivated plants are likely to be somewhat different in their properties from those gathered from the natural habitats, it is also clear that certain values in plants can be deliberately enhanced under controlled condition of cultivation. This is a very crucial meeting point of traditional herbal medicine and biomedicine at which difficulty of 'togetherness' somehow emerges. Most standard pharmacological evaluations of medicinal plants tend to support the use of traditional medicines but fall short of suggesting that efficacy must be culturally appropriate. Scientific clinical trials are supposed to provide 'proof' of efficacy and allow these medicines to be admitted into evidence-based medicines.

However, from the discussion in the earlier part of this section, what works in traditional herbal medicines would seem to include transcendent, transformational 
experiences, changing lived-body experience and the gaining of meaning. Traditional medical practitioners have in fact been referred to as "excellent psychologists" (Crawford and Lipsege, 2004) in that their therapy is so widely regarded as successful because psychosocial issues are addressed and as such the "meanings of sicknesss events become a social issue" (Goody, 1977) thus interpreted as structural microcosms of the society as a whole (Swedlund and Armelagos, 1990). Of what use, therefore, is the clinical trials of herbal medicines? Apart from the efficacy and safety issue, some have suggested that there are practical benefits because biomedical criteria can generate valuable new interpretations for comparative studies of human physiological processes, the ways in which such processes are perceived and the culture-specific behaviours that these perceptions produce. An example of this point would be Browner, Ortiz de Montellano and Rubel, (1988: 683). These authors developed a model for analyzing ethnomedical data independently as well as according to the standards of biomedicine.

The first step in their model is to identify the phenomena under investigation in emic terms. In the second step, one determines the extent to which the phenomena described can be understood in terms of biomedical concepts and methods. The third and final step is to identify the area of convergence and divergence between the emically described phenomena and their biomedical understandings. At this stage, biomedical concepts are used not to examine the phenomena in their own terms (as in the second step) but to see if they are consistent with biomedical assumptions. Etkin (1988) suggested how efficacy can be measured culturally in what is a seminal piece in this regard. He defines efficacy as "something that works by directly producing a set of required, culturally defined outcomes" (p30) and suggested that the key to any consideration of efficacy is the distinction between its emic and etic interpretations. The efficacy of medicinal plants may be judged in their ability to induce full remission of symptoms. One notes this particular point in the conversations with traditional healers on the UWC-PEPFAR project. The efficacy of medicinal plants is judged not so much because they have been in use for generations but their ability to induce full remission of symptoms. Physical signs such as fever salivation are important indicators that a plant has initiated the healing process.

Ortiz de Montellano and Browner (1985) both developed a method for assessing the efficacy of medicinal plants according to both indigenous understanding of their therapeutic effects and the standards of biomedicine. The first step in their method requires investigating informants' own understandings of illness aetiology to predict what medicines would be considered appropriate for their treatment. These ethnomedical data are then combined with data on chemical constituents, physiological effects and biomedical concepts that constitute 'confidence levels' for the efficacy of plant medicines. Level I comprise reported folk use. Multiple reports of use by populations widely dispersed through space, or persistent reports over long periods of time, increase the probability that a plant will exhibit pharmacological activity. Level II plants meet the 
criteria of Level I and show the desired activity of the isolated compounds or extracts in in vitro or in vivo tests. At Level III, plants satisfy Level II requirements and show a plausible biochemical mechanism by which the active constituents could exert the indicated physiological effect. Finally Level IV plants fulfill the criteria for Level III and have been clinically tested, or are commonly used in medicine. Information on a plant's level of confidence is then considered in the light of its emic evaluation of efficacy. This method would seem to go the distance in terms of understanding of the therapeutic effects of medicinal plants, but in practice, are clinical trials conducted along what is suggested? For this, we examine the clinical trial of a herbal plant using one of such trials at the South African Herbal Science and Medicine Institute (SAHSMI).

\section{SAHSMI and the Clinical Trial of Sutherlandia}

The South African Herbal Science and Medicine Institute (SAHSMI) based at the University of the Western Cape in Cape Town conducted what could be considered the first randomised double-blind, placebo-controlled trial of sutherlandia in healthy adults in the country. The Institute which houses the International Centre on Indigenous Phytotherapy Studies (TICIPS), a global centre on indigenous medicine funded by the National Centre for Complementary and Alternative Medicine (NCCAM) of the National Institutes of Health (NIH) in Washington, DC, USA., conducts clinical trials of herbal plants in collaboration with the School of Medicine at the University of Missouri in the US, the Nelson Mandela Medical School of the University of KwazuluNatal in Durban, South Africa, the Institute for Infectious Diseases and Molecular Medicine (IIDMM) of the University of Cape Town in South Africa, the Medical Research Council (MRC) of South Africa. This collaborative endeavour is rooted in biomedical culture in that what is sought in the clinical trials are evidence for efficacy and safety. Such evidence is informed by the idea that building a valid basis for knowledge in herbal medicine will require balancing two aspects of scientific validity: internal and external validity (Cravotto; Boffa; Genzini and Garells, 2010). Internal validity means that the research must reliably test hypothesized relationships between an intervention and outcome under controlled conditions. Internally valid research will typically try to answer a focused research question that is salient within the vocabulary and a method of scientific community at the time the research is conducted. External validity refers to the applicability of the research results to a target population outside the experimental conditions of the research study. External validity must always be weighed against the need for rigorous internally valid research.

The clinical trial of sutherlandia, a shrub that belongs to the Fabeca family, at SAHSMI was along this line (see Quinton et al 2007). Surtherlandia is principally constituted of L.-canavanine, a non-protein amino acid. Gericke et al (2001) note that there is about $30-40 \mathrm{mg}$ of L-canavanine per dry gram of the $S$. frutescens leaf. Other principal 
constituents are GABA, and D-pinitol. The latter is a type of sugar 'found in many types of legumes and is classified as a chiro-inositol. It is also known as 3-O-methylD-chiro-inosotol, or 3-0-methyl-1,2,4 cis-3,5,6 trans-hexahydroxyl-cyclohexanol (Mills et al,2005) GABA (gabba-amino butyric acid) is both an amino acid and inhibitory neurotransmitter. It is found at levels of $14 \mathrm{mg}$ per gram dry leaf of $S$. frutescens (Gerike et al, 2001). Sutherlandia is indigenous to South Africa and is widely used in the country as traditional medicines "since they were first adopted by the Khoi, San, and Nama peoples" (Johnson et al, 2007:0002). Like most traditional herbal medicines, its usage is not confined to the treatment of a single illness but several illnesses such as cancer, tuberculosis, diabetes, influenza, rheumatoid arthritis, osteoarthritis, peptic ulcer and in the current era of HIV/AIDs, some of the associated symptoms like nausea (Gericke et al, 2001; Dalvi, 2003; Johnson et al, 2007). The various names given to it by different ethnic groups in the country reflects its wide usage. We are told that it is referred to as Unwele, Lerumo-lamadi, Unwele, kankerbos, kankerbossie (Mills et al, 2005 citing Gericke et al, 2001; Dalvi et al 2003). Sutherlandia does not have any rules that govern its use like most herbal plants that are widely sold in the country. Consequently, the absence of any form of regulation has meant that the harmonization of its use could also be considered as an issue. Its importance for clinical trials lies, partly, in this.

The objectives of the clinical trials at SAHSMI were twofold: to conduct a pilot study of the safety of sutherlandia in healthy adults and secondly, to contribute to establishing procedures for ethical and scientifically \{emphasis mine\} rigorous clinical trials of African indigenous medicines. The design was a randomised, double-blind placebo-controlled trial of sutherlandia leaf: 12 participants were randomised to a treatment of $400 \mathrm{mg}$ capsules of sutherlandia leaf powder, 13 individuals were randomised to consume a placebo capsule, each receiving 180 capsules for the 3 months duration of the trial which was at the Tiervlei Trial Centre of the Karl Bremer Hospital in Bellville, Cape Town, South Africa. Clearly, what we see here is that when medicinal plants with biologically active constituents are the focus of study, biomedical researchers fail to consider the cultural contexts within which plants are used with the result that efficacy of a plant is misunderstood (Walstein and Adams, 2006). Can one say this of the scientists at SAHSMI. The scientists at SAHSMI will probably argue that they are aware of the cultural context and that given the challenges posed by the 'medication of HIV/AIDs crisis', effective crisis intervention is dependent on accurate assessment that directly translates into focusing treatment when it is needed. They will go on to point out that the philosophical basis of clinical trials at the Institute is not just about providing the evidence of the efficacy and safety of traditional medicines, rather, it is about enhancing the well-being of the patients' safety and cure of disease, which, keen observers would not hesitate to point out that, though commendable, is hardly different from what biomedical researchers would generally say. The scientists know very well that traditional as well as biomedicine is integral 
components of the health care delivery system in the country and indeed, in several African countries. One can suggest that that they will also know that the individual's interaction with other sectors of health care depends on how the health problem is perceived or sanctioned by the sick person and most importantly by relatives and close friends as shown by Crawford and Lipsege's exploratory study of the identification of psychological distress among the Zulu people in South Africa (2004). Depending upon the prevalent symptoms of illness, a person may choose to disregard an illness or heath problem, use treatment modalities known to the individual, friends or family or make decision to use services of a traditional medical practitioner or biomedical health practitioner (Tabi et al, 2006). Despite the 'cultural awareness' of the scientists at SAHSMI, there is still the difficulty of going beyond the narrow concern, as exemplified by clinical trials, with safety, efficacy and toxity of plants to a focus on how people use their knowledge of such plants - the uses to which people put their knowledge of the natural world and classification as it relates to their exploitation of it. This perhaps explains why SAHSMI sought anthropology and sociology in it's broad interdisciplinary understanding of herbal medicines and clinical trials.

\section{Concluding Remark: Biomedical hegemony?}

Biomedicine is charged in the wider critique of the continuing hegemonic domination of the West over all aspects of life outside the western world. This is not without foundation. In a penetrating piece on applied ethnobiology, Sillitoe (2006) notes that "the term ethnobiology did not come into use until the twentieth century. Naturalists have shown an 'interest' in local biological knowledge since the time Europeans started to explore the world from the fifteenth century onwards. Indeed this knowledge informed biological science from early on, as witnessed in the research by Linnaeus, who corresponded with people around the world, and Rumphius' work in Southeast Asia. In a sense, we can interpret this as ..... Europeans not only sought to understand the new regions they invaded but also were on the lookout for resources that they might profitably exploit engaging practices that today we should consider tantamount to biopiracy......... Those who engage in bio-prospecting, hoping for example, to find elsewhere plants with unknown medicinal or cosmetic properties, are also current-day descendants of this tradition, although from a humanistic perspective such work gives rise to worries about theft of others' knowledge". Would this explain the non-availability of the traditional healers' knowledge for scientific inquiry and its concomitant dismissal that it does not have scientific legitimacy?

It is very clear in the vast literature on traditional healing that in the 'clinical setting' of traditional healing, the encounter between the traditional healer and the patient is not necessarily between the two: both ancestors are involved. The clinical reasoning of the traditional healer is thus a multilayered process of the interaction in the clinical 
setting which reveals to the traditional healer certain 'signs' of which the bodily signs is a part and interpretations thereof, as well as the consultations with the ancestors for the understanding of the patient's 'disequilibrium' and what to do to bring it back to the state of 'equilibrium'. This epistemological complexity of understanding shows that what is 'prescribed' is specific to the patient as an individual person, not as a body and as such would hardly yield itself to the efficacy sought through clinical trials that are controlled, measured and regarded as valid. Traditional medical healing modality, one must emphasise, has withstood the test of time but, perhaps, not the test of science, which is still grappling with the mind-body interconnections. In the traditional healers' view, clinical trials may yield an understanding of the herbs but this is not equivalent to the understanding of the patient's state of 'person-disequilibrium'. To them, this cannot be ignored when talking about efficacy of herbs.

\section{Acknowledgement}

This paper, as mentioned in the text, is an outgrowth of an ongoing project exploring the philosophy and environment of clinical trials of herbal plants at the South African Herbal Science and Institute (SAHSMI) at the University of the Western Cape. Some of the materials used for the discussion derive from my interaction with staff and students of the Institute as well as from a tape-recorded interview with the Director of the Institute, Prof Quinton Johnson.

\section{References}

Ang-Lee, M. K., Moss, J., and Yuan, C. S., 2001 Herbal medicines and perioperative care JAMA 286: 208-16

Bent, S., 2008 Herbal medicine in the United States: review of efficacy, safety and regulation - grand rounds at the University of California, San Francisco Medical Centre Journal of General Intern Medicine 23: 854-9

Browner, C. H., Ortiz de Montellano and Rubel, 1988 A methodology for cross-cultural Ethnomedical Research Current Anthropology 29, 5

Calapai, Gioacchino, 2008 European Legislation on Herbal Medicines Drug Safety 31, 5:428-431

Calapai, G. and Caputi, A. P. 2007 Herbal medicines: can we do without pharmacologists? Evidence Based Complementary Alternative Medicine 4:41-3

Calixto, J. B., 2000 Efficacy, safety, quality control, marketing and regulatory guidelines for herbal medicines (phytotherapeutic agents) Brazilian Journal of Medical Biological Research 33, 2:179-89 
Coultier, I. and Willis, E., 2004 The rise and rise of complementary and alternative medicine: A sociological perspective Medical Journal of Australia 180: 587-589

Cravotto. G., L. Boffa, L. Genzini and D. Garella, 2010 Phytotherapeutics: an evaluation of 1000 planta Journal of Clinical Pharmacy and Therapeutics 35:11-48

Crawford, T. A. and Lipsege, M., 2004 Seeking help for psychological distress: The interface of Zulu traditionasl healing and Western biomedicine. Mental Health, Religion and Culture, 7, 2:131-148

Dalvi, S., 2003 Surtherlandia for immune support Positive Health 2003:23-25

De Smet, PAGM., 2002 Herbal remedies. New England Journal of Medicine 347: 2046-2056

Ernst, E., 2002 Adulturation of Chinese herbal medicines with synthetic drugs: a systematic review Journal of International Medicine, 252:107-13

Etkin, N. C., 1988 Ethnopharmacology: Biobehavioral approaches in the Anthropological study of indigenous medicines Annual Review of Anthropology Vol 17:23-42

Fabrega, H., 2002 Medical validity in eastern and western traditions. Perspect Biological Medicine 45: 395-415

Forster, G. M., 1976 Disease etiologies in Non-Western Medical Systems American Anthropologist 78:773-82

Gericke, N., Albrecht C. F., Van Wyk B. Mayeng B, Mutwa C, Hutchings A., 2001Sutherlandia frutescens. Australian Journal of Medical Herbalism 13:9-15

Goody, Jack, 1977 Domestication of the Savage Mind Cambridge: Cambridge University Press

Johnson, Q. Syce, J., Well, H., Rudeen, K., and Folk, W.R., 2007 A Randomised, Double-Blind, Placebo-Controlled Lessertia Frutescens in healthy adults PLOS Clinical Trials

Kaptchuck, T. J., 2001 The double-blind, randomized placebo-controlled trial: gold standard or golden calf? Journal of Clinical Epidemiology 54, 541-9

Mackenzie-Cook, Peter, D., 2006 Challenging the New Orthodoxy in Integrative Medicine The Journal of Alternative And Complementary Medicine Vol12, 7:679-683

Mahady, G. B., 2001Global harmonization of herbal health claims Journal of Nutrition 131 (3 supplementary): 1120-1123S

Mills, S. E., 2005 HIV Illness meaning and collaborative healing strategies in South Africa Social Dynamics 31 2:126-160

Mills, Edward, Curtis Cooper, Dugald Seely and Izzy Kanfer, 2005 African herbal medicines in the treatment of HIV: Hypoxis and Sutherlandia. An overview of evidence and pharmacology Nutritional Journal 4, 19 available (http:// 
creativecommons.org/licenses/by/2.0)

Montogomerry, K., 2006 How doctors think: clinical judgement and the practice of medicine Oxford: Oxford University Press

Morris, K., 2002 South Africa tests traditional medicines. Lancet Infectious Disease 2:319

Nebel, A., Schneider, B. J. Baker, R. K. et al, 1999 Potential metabolic reaction between St John's wort and theophylline [letter] Annal Pharmacother 33:502

Nyika, A. 2007 Ethical and Regulatory Issues surrounding African Traditional

Medicine in the Context of HIV/AIDS, Developing World Bioethics 1: 2534

Oloyede, Y., 1997 Pain in Hausa and Western culture: a comparison. Unpublished paper.

Department of Psychiatry, Royal Free and University College Medical School, University College, London

Ortiz de Montellano and Browner, C. H., 1985 Chemical basis for medicinal plant use in Oaxaca, Mexico Journal of Ethnopharmacology Vol. 13; 57-88

Pefile, S, 2005 South African legislation on traditional medicine. Science and

Development Network

Prost, N., Tichadou, L., Rodor, F. et al, 2000 St John's wort venlafaxine interaction Presse Med 29:1285-6

Protorious, E., de Klerk, G. W. and van Rensburg, H. C. J., 1993 The traditional healer in South African health care Pretoria: Human Sciences Research Council

Rubin, A., 1989 Art as Technology In: The Arts of Africa, Oceania, Native Americas. (ed.) Z. Pearstone. Beverly Hills

Schaffner, K. F., 2002 Assessments of efficacy in biomedicine: the turn toward methodological pluralism. In: Callahan, D. (ed.) The role of complementary and alternative medicine: accommodating pluralism. Washington, D.C.:

Georgetown University Press

Sillitoe, Paul., 2006 Ethnobiology and applied anthropology: reapprochment of the academic with the poractical Journal of the Royal Anthropological Institute (NS) pp. 119-142

Stevens, P. Jr., 1996 Magic In Encyclopedia of Cultural Anthropology (eds.) D.

Levinson and Ember, pp.721-26

Stickel, F., Patseuker, E., and Schuppan, D., 2005 Herbal Hepatotoxicity Journal of Hapatology August pp.901-910

Swedlund, A. C., Armelagos, G. (eds.), 1990 Disease in Populations in transition: anthropological and epidemiological perspectives New York: Bergen and Garvey P.

Tabi, Z., Lynch, F., Ceredig, R., Allan, J. E. and Doherty, C., 1988 Virus specific memory T cells are Pgp-1+ and can be selectively activated with phorbolester and calcium Cellular Immunology Vol 113 issue 2:268-277 
Taussig, M., 1992 Tactility and Distraction In Rereading Cultural Antbropology, (ed.) G. E. Marcus pp. 8-14 Durham

Tilburt, Jon C., and Kaptchuck, Ted, J., 2008 Herbal medicine research and global health: an ethical analysis Bulletin of the World Health Organization, August, 86, 8. I8b

Waldstein, A. and Adams, C., 2006 The interface between medical anthropology and medical ethnobiology Journal of the Royal Anthropological Institute Vol 12, pp. $95-118$

WHO, 2002 WHO traditional medicine strategy 2002-2005, Geneva: WHO

Wolff, Norma. H., 2000 The Use of human Images in Yoruba Medicines Ethnology, Vol. 39, no 3: 205-224

\section{End Notes}

1 The traditional medicine system in Africa includes herbal remedies for specific diseases, folk knowledge, traditions and values, health behaviours and patterns.

2 The UWC-PEPFAR-CDC Project 7 is specifically concerned with the training of traditional healers and biomedical health professionals in dealing with HIV/AIDS. It is an attempt to bring together both health practitioners within the context of a wider science-traditional medicine paradigms engagement/meeting. 